\title{
Finding Optimum Wavelet Decomposition Level Using Autocorrelation
}

\author{
Omar Salah F. Shareef ${ }^{1}$, Soong Der Chen ${ }^{2}$ \\ ${ }^{1}$ University of Fallujah, Fallujah, Iraq \\ omar.alshareef@uofallujah.edu.iq \\ ${ }^{2}$ College of Information Technology, Universiti Tenaga Nasional, Malaysia \\ Chensoong@uniten.edu.my
}

\begin{abstract}
Wavelet transform is used to carry out time-frequency analysis including filtering of noises and reconstruction of the signal. Wavelet decomposition at a low level for denoising of signal is not very impactful in reducing noise. Alternatively, a high-level of wavelet decomposition may eliminate useful information carried by the signal. This study hinges on finding the optimum level of wavelet decomposition when performing denoising using wavelet transform. Optimum decomposition level is estimated based on parameters: mean and autocorrelation of residual in wavelet denoising. The importance of the paper is significantly the reduction of noise in the received signals whilst maintaining sufficient signal quality to allow the information carried by the signal to be read.
\end{abstract}

Key words: Optimum level, Wavelet Decomposition, Denoising, Characteristics of AGWN, Autocorrelation, and SNR.

\section{INTRODUCTION}

The researchers in the arena of the wavelet field feel that by using wavelets, one is adopting a whole new mindset or perspective in processing data in signal processing and image processing for the advancement of information technology.

Modulation technique is typically implemented by using a Fourier transform. The use of Fourier transform in conventional signal processing is bound to uncover the information of either the frequency domain or time domain of the modulated signal [1]. Fourier transform is not able to yield a satisfactory result in denoising the received signal.

Wavelet transform shows better performance with time-frequency analysis to filter noise with the appropriate choice of wavelet decomposition level [2, 3, 4].

In the modulation technique, the performance is increased with time-frequency analysis simultaneously with wavelet transform [5,6]. The reduction of noise can be optimized with the use of wavelet transform $[7,13]$. There has also been some
FFT [8,9]. However, the current use of DWT only performssingle-level wavelet decomposition when performing denoising. Although wavelet decomposition with higher level is better for noise reduction, it may also yield an unreadable signal $[10,11]$. On the other hand, low decomposition level may not be sufficient to reduce noise effectively from the received signal. Therefore, it is necessary to find the optimum wavelet decomposition level for denoising. Simulation with Matlab wavelet tools will be conducted to attain the optimum level of using wavelet decomposition for denoising.

\section{METHODOLOGY}

In the research work, optimum wavelet decomposition level among different levels is obtained with the wavelet analysis using Matlab 2009. Sinusoidal signal with different frequencies is used as a reference signal in the analysis.

AWGN is added with the reference signal through random data generation in our simulation programming. Optimum decomposition level is obtained by measuring the criteria of mean value and autocorrelation of AWGN.

This estimated optimum level is compared with the actual optimum level to demonstrate the performance of the algorithm. The actual optimum level is obtained according to the SNR of reference signal.

In the optimum outcome, the denoised signal should be identical to the reference signal. With the use of an optimal decomposition level, there would be more reduction of noise and a lesser amount of reduction of signal information in the denoised signal. Figure 1 shows the flowchart of the proposed model as follow. 
Omar Salah F. Shareef et al ., International Journal of Advances in Computer Science and Technology, 8(9), September 2019,27 - 31

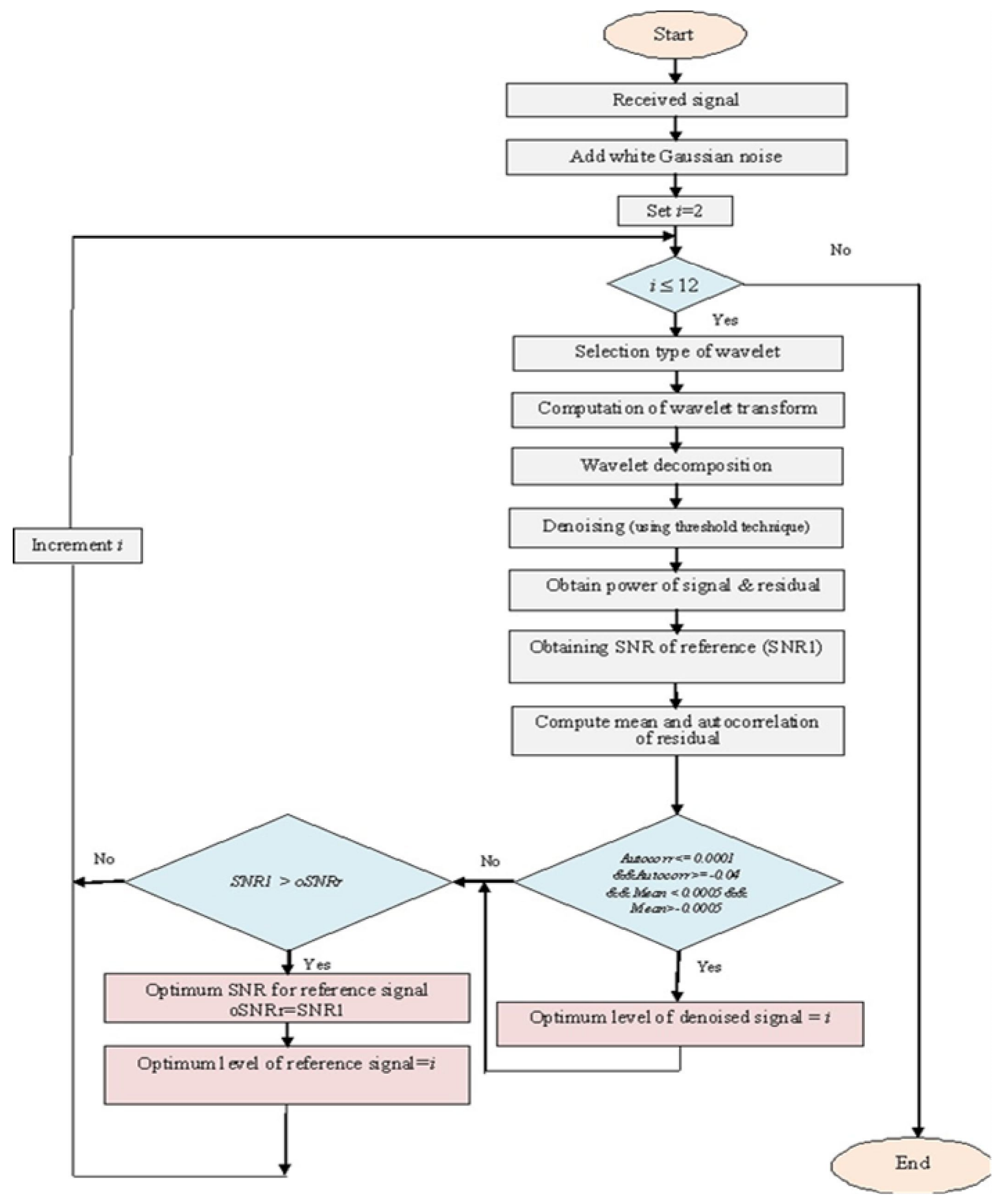

Figure 1: Model of Finding Optimum Wavelet Decomposition 


\subsection{Mean of AWGN}

AWGN is usually used to depict the background noise of the signal. The noise amplitude has a Gaussian distribution.

The mean is particularly used to describe the density function. For AWGN, the average value, or mean, equal zero. The Gaussian distribution is an important probability distribution. The standard normal distribution is the normal distribution with zero means $(\mu=0)$.

\subsection{Autocorrelation of AWGN}

Autocorrelation is an important factor in a time series evaluation. Autocorrelation coefficients are a series of quantities, which measure the correlation among observations at different times [14].

As the characteristics of AGWN, it shows mean value and autocorrelation are zero. When the signal is denoising, for some level, the signal part is also ditched in the denoised signal, this ditched signal portion is included in residual. That is why we should check up the residual included not only AWGN but also some portion of signal. If we check out the mean value and autocorrelation of residuals, we can then be confirmed whether there is only AWGN or AWGN adding with some signal portion, because if there is only AWGN the mean value and autocorrelation should be around zero.

For the autocorrelation of AWGN, the value of autocorrelation function (acf) would be 1 at lag 0 (index is 1). The other positions of the lag index show the acf value as 0 . FFT of this autocorrelation function would be the same for each lag position as energy spectrum versus frequency representation.

Thus, the statistical parameter is justified whether signal information is including with AWGN in residual or not. It is demonstrated as a practical experiment, low-level wavelet decomposition shows that the AWGN is fully in the residual. On the other hand, high-level wavelet decomposition also shows there is some signal information merged with AWGN in residual.

Figure 2(a,b) illustrates the matching of the original and denoised signal and the statistics of the residual signal after denoising. The statistics show the mean value (histogram) and the autocorrelation of residuals. If there is only AWGN in residual, the histogram should follow the characteristics of Gaussian distribution where mean value is 0 .

Figure 2(a) shows an example of good denoising. The denoised signal shows good matching when compared to the original signal. Figure 2(b) shows an example of poor denoising.

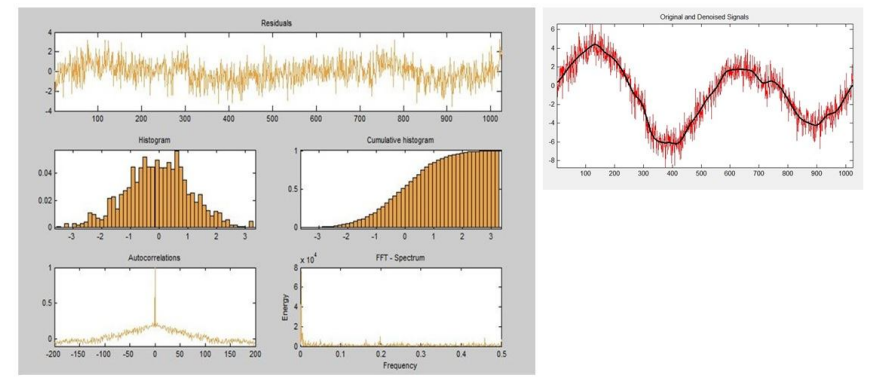

(a)

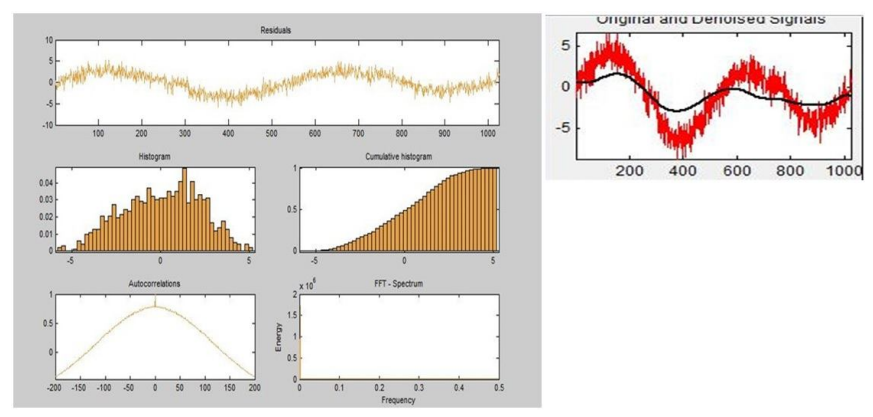

(b)

Figure 2:

(a). Example of Efficient Denoising

(b). Example of Poor Denoising

\subsection{Signal-to-Noise Ratio (SNR)}

Signal-to-noise ratio generically indicates the dimensionless ratio of the signal power to the noise power contained in a recording [12]. SNR parameterizes the performance of optimal signal processing systems when the noise is Gaussian.

The signal-to-noise ratio is typically written as SNR and equals:

$$
S N R=\frac{P_{S}}{P_{N}}
$$

Where, $P_{\Upsilon}$ is considered as the average power of the signal and $P_{N}$ as the average power of noises.

The estimated optimum level is compared with the actual optimum level to evaluate the optimality of that particular decomposition level. In obtaining the actual optimum level, the reference signal is used. Residual is taken as the difference between the reference signal and the denoised signal. SNR for actual decomposition level is estimated through the ratio of the parameter of power of denoised signal and power of the residual following the wavelet denoising.

\section{EXPERIMENTS AND RESULT}

The range of decomposition levels which are utilized in our experiments is as 2 to maximum allowed decomposition level. 
In this experiment, 12 is the maximum possible level considering the 32000 samples of the reference signal. Where very low frequency of the Sinusoidal signal is obtained through $\sin \left(0.0625^{*} \mathrm{t}\right)$ and very high frequency are obtained in terms of $\sin (8 * t)$ in obtaining the optimum level of wavelet decomposition. In the experiments, three types of wavelet as Daubechies, Coiflet, and Symlet are harnessed to obtaining optimum level of wavelet decomposition.

In an efficient denoising scheme, the denoised signal should be closer to the reference signal. Also, the experiments have been conducted to evaluate the result of the optimum level of wavelet decomposition with autocorrelation and mean separately.

Table 1, 2 and 3 shows the comparison of the actual optimum level, the optimum level with autocorrelation, the optimum level with mean value and the estimated optimum level with both autocorrelation and mean value of $\mathrm{db} 2$, coif2, and sym 2 wavelet.

Table 1: Comparison of the Optimum level for $\mathrm{db} 2$.

\begin{tabular}{|c|c|c|c|c|}
\hline Signial & Actual level & Autocomelation & Mein & $\begin{array}{l}\text { Awtoconelation } \\
\& \text { mean }\end{array}$ \\
\hline $\sin \left[0.0623^{\circ} t\right]$ & 9 & 9 & 9 & 9 \\
\hline $\sin \left(0.125^{*} t\right)$ & 8 & 8 & 12 & 8 \\
\hline $\sin (0.25+1)$ & 5 & 8 & 7 & 8 \\
\hline $\sin \left(05^{2} t\right.$ & 7 & 7 & 11 & 7 \\
\hline $\sin \left(1^{*} \mathrm{t}\right)$ & 6 & 6 & 11 & 6 \\
\hline $\sin \left(2^{2} t\right)$ & 5 & 5 & 11 & 5 \\
\hline $\sin \left(4^{x} t\right)$ & 4 & 5 & 9 & 5 \\
\hline $\sin \left(8^{2} t\right)$ & 4 & 4 & 8 & 4 \\
\hline
\end{tabular}

Table 2: Comparison of the Optimum level for coif2.

\begin{tabular}{|c|c|c|c|c|}
\hline Siznal & Actual level & Autscorralation & Mean & $\begin{array}{l}\text { Autocorrelation } \\
\text { \&s mean }\end{array}$ \\
\hline $\sin (0,0425 * 1)$ & 50 & 10 & 8 & 9 \\
\hline $\operatorname{sen}\{0.175)^{\circ}$ & ${ }^{3}$ & B & 12 & 3 \\
\hline $\sin \left(0,25^{\circ}\right)$ & 8 & 8 & 12 & 8 \\
\hline $\sin (0.54)$ & 8 & 8 & 9 & 7 \\
\hline $\sin (i+\pi)$ & 7 & 7 & 30 & 7 \\
\hline $\sin \left(x^{2} \theta\right)$ & 6 & 6 & 10 & 6 \\
\hline $\operatorname{sen}\left(9^{\circ} \theta\right)$ & 5 & 5 & 7 & 5 \\
\hline $\sin (8+\theta)$ & 4 & 4 & 8 & 4 \\
\hline
\end{tabular}

Table 3: Comparison of the Optimum level for sym2.

\begin{tabular}{|c|c|c|c|c|}
\hline Signal & Actaal level & Autcontelation & Mean & $\begin{array}{l}\text { Autocomelation } \\
\text { \&f mean }\end{array}$ \\
\hline $\sin \left(00625^{\circ}\right)$ & 9 & क & 8 & 8 \\
\hline $\sin \left(0.125^{*}\right)$ & 8 & 9 & 11 & 8 \\
\hline $\sin \left(0.5^{\circ} b\right.$ & 8 & 8 & 10 & $\$$ \\
\hline mosti) & 7 & 7 & $\mathrm{~s}$ & 7 \\
\hline $\sin \left(1^{2}\right)$ & 6 & 6 & 12 & 6 \\
\hline और $\left.z^{-1}\right)$ & 5 & 5 & 8 & 3 \\
\hline $\sin \left(4^{-1}\right)$ & 4 & 5 & 10 & 5 \\
\hline $\sin \left(8^{-1}\right)$ & 4 & 4 & $\mathrm{~g}$ & 4 \\
\hline
\end{tabular}

The denoised signal that is obtained using the optimum level shows a good fit when compared with the reference signal. The result of the experiments shows the performance of the autocorrelation only, mean only and all together. The small variation is shown sometimes in the results of experiments with autocorrelation.

The results show that for autocorrelation only and both are the same optimum level decomposition with the actual level. However, the autocorrelation can meticulously foresee the actual optimum level. For mean only, the results show the poor prediction.

\section{CONCLUSION}

Wavelet transform is more preferable for conducting time and frequency domain analysis simultaneously. With wavelet decomposition, noisy received signals can be denoised at the receiver. High level of wavelet decomposition demonstrates maximum reduction of noise in signal denoising. However, there is a potential to miss information carried by the signal. Low level of wavelet decomposition is not sufficient to filter all of noises from the signal.

The performance of the optimum level of wavelet decomposition is based on the autocorrelation of AWGN, which can correctly predict the actual optimum decomposition level across different frequencies and wavelet modes.

\section{REFERENCES}

1. C.S. Burrus and T.W. Parks, DFT/FFT and Convolution Algorithm, John Wiley \& Sons, New York, 1985 
2. H. Zhang, D. Yuan, M. Pätzold, Novel study on PAPRs reduction in wavelet-based multicarrier modulation systems, Digital Signal Process. Vol. 17, pp. 272-279 https://doi.org/10.1016/j.dsp.2006.08.002

3. S. Rajbhandaril, Z. Ghassemlooy, M. Angelova, A Study of Discrete Wavelet Transform Based Denoising to Reduce the Effect of Artificial Light Interferences for Indoor Optical Wireless Communication, OWC-19 610 CSNDSP, 2010.

4. S. Rajbhandari, Z. Ghassemlooy, and M. Angelova, Effective denoising and adaptive equalization of indoor optical wireless channel with artificial light using the discrete wavelet transform and artificial neural network, IEEE-Journal of Lightwave Technology, vol. 27, pp. 4493-4500, 2009. https://doi.org/10.1109/JLT.2009.2024432

5. M. You and J. Ilow, A Multi-Wavelet Packet Modulation In Wireless Communications, CCECE 2004- CCGEI 2004, Niagara Falls, Maylmai, IEEE, 2004.

6. S. I. Hassouna, E. F. Badran, D. A. Mohamed and S. Shaaban, Wavelet Packet Modulation for Multi-carrier CDMA Communications, 24th National Radio Science Conference (NRSC 2007). https://doi.org/10.1109/NRSC.2007.371385

7. Omar, S.F., Salman Yussof and Islam T. Finding the Optimum Level of Wavelet Decomposition for Reducing Noise in Wireless Communication, vol. 5, no.11, pp. 1212-1217, 2011.

8. L. F. Q. Silveira, L. G. Q. Silveira Junior, F. M. Assis, and E. L. Pinto, Analysis and Optimization of Wavelet-Coded Communication Systems, IEEE Transactions on Wireless Communications, vol. 8, no. 2, February 2009.

https://doi.org/10.1109/TWC.2009.070978

9. M. Gautier and J. Lienard. Performances of complex wavelet packet based multicarrier transmission through double dispersive channel. NORSIG 06 IEEE Nordic Signal Processing Symposium (Iceland), June 2006. https://doi.org/10.1109/NORSIG.2006.275280

10. M. Gautier, M. Arndt, J. Lienard. "Efficient wavelet packet modulation for wireless communication," The Third Advanced International Conference on Telecommunications, AICT 2007, May 13-19, 2007 -Mauritius.

11. A. C. Bingham, "Multicarrier modulation for data transmission: An Idea Whose Time Has Come," IEEE Communications Magazine, vol. 28, no.5, pp. 5-14, 1990.

https://doi.org/10.1109/35.54342

12. John A.C. Bingham, ADSL, VDSL and Multicarrier Modulation. 2000 Johan Wiley \& Sons, Inc., NY. https://doi.org/10.1002/0471200727

13. Chakraborty S. Bandyopadhyay S.K. Emerging Biometric Technology-A Review. International Journal of Advances in Computer Science and Technology. 2016; 5(1): 8- 22.

14. Maynard \& Triyanti V. Evaluation Of The Correlation Between Hand Anthropometry And Grip Strength in Sedentary Undergraduate Students. International Journal of Advances in Computer Science and Technology. 2016; 5(3): 38- 46. 